\title{
Estructura e invarianza factorial del Inventario de asertividad de Gambrill y Richey en adolescentes peruanos
}

\author{
Structure and factorial invariance of the Gambrill and Richey Assertiveness \\ Inventory in Peruvian adolescents
}

\author{
Cristian Ramos-Vera ${ }^{1}$ \\ Sociedad Peruana de Psicometría \\ Evalia Sulma Cahuana Moroco², Gloria Maribel Juárez Jurupe ${ }^{3}$, \\ Universidad Cesar Vallejo \\ Vladimir Illich Navarro Vargas ${ }^{4}$ \\ CPT. Desarrollo Personal
}

Recibido: $11-05-21$

Aceptado: $30-08-21$

Publicado: $23-12-21$

\begin{abstract}
Resumen
El presente estudio tiene como objetivo evaluar la estructura factorial y la invariancia de medida según el sexo del Inventario de Asertividad de Gambrill y Richey (1975) en adolescentes peruanos compuesto por dos subescalas de Grado de Incomodidad e Índice de Respuesta, se utilizó una muestra no probabilística de 639 adolescentes peruanos de 14 a 20 años $(M=$ $15.84, D E=0.86$ ). Se obtuvo evidencias de validez basado en el criterio de jueces como parte del proceso de adaptación contextual y lingüística para la población evaluada. Se realizó el análisis factorial confirmatorio de 3 modelos de la subescala de grado de incomodidad (GI) y 3 modelos en la subescala de Índice de Respuesta (PR). Posteriormente se evaluó la equivalencia de medición de ambas subescalas evidenciando que la presente medida es invariante según sexo en adolescentes peruanos.
\end{abstract}

Palabras clave: Asertividad; adolescentes; psicometría; validez.

\section{Abstract}

The present study aims to evaluate the factorial structure and measurement invariance according to sex of the Assertiveness Inventory of Gambrill and Richey (1975) in Peruvian adolescents composed of two subscales of Degree of Discomfort and Response Index, using a non-probabilistic sample of 639 Peruvian adolescents aged 14 to 20 years $(M E=15.84$,

\footnotetext{
Autor para correspondencia: cristony_777@hotmail.com ORCID: https://orcid.org/0000-0002-3417-5701

2 E-mail: cahuana703@gmail.com ORCID: https://orcid.org/0000-0001-6372-5760

3 E-mail: goyita2911@gmail.com ORCID: https://orcid.org/0000-0002-1413-8460

4 E-mail: vladimirnavarrocpt1982@gmail.com ORCID: https://orcid.org/0000-0002-3246-9263
}

(C) Los autores. Este artículo es publicado por la Revista de Investigación en Psicología de la Facultad de Psicología, Universidad Nacional Mayor de San Marcos. Este es un artículo de acceso abierto, distribuido bajo los términos de la licencia Creative Commons Atribución 4.0 Internacional (CC BY 4.0) [https://creativecommons.org/licenses/by/4.0/deed.es] que permite el uso, distribución y reproducción en cualquier medio, siempre que la obra original sea debidamente citada de su fuente original. 
$S D=0.86$ ). Evidence of validity was obtained based on the criterion of judges as part of the contextual and linguistic adaptation process for the evaluated population. Confirmatory factor analysis was performed on 3 models of the degree of discomfort (GI) subscale and 3 models on the Response Rate (RP) subscale. Subsequently, the equivalence of measurement of both subscales was evaluated, showing that this measure is invariant according to sex in Peruvian adolescents.

Keywords: Assertiveness; adolescents; psychometrics; validity. 
La asertividad es una habilidad esencial en la vida de los adolescentes en la escuela, pues permite la honesta expresión de los sentimientos de manera correcta (Coronel, Levin y Mejail, 2011) y la aceptación de las reglas (Pailos, 2014). Esta conducta es favorable en el ámbito socioeducativo ya que puede marcar diferencias entre el éxito y el fracaso escolar (Domínguez, López \& Álvarez, 2015).

La capacidad asertiva es un tema de repercusión actual en la investigación psicológica debido a la influencia en los adolescentes en temas tales como la autoestima (León, Rodríguez, Ferrel \& Ceballos, 2009), rendimiento académico (González, Guevara, Jiménez y Alcázar, 2018), empatía y las relaciones sociales (García-López, Díaz, González-Víllora y Valenzuela, 2012), motivación académica (Sitota, 2018), socialización parental (Espinoza \& Panta, 2014). Así también, la falta de asertividad puede estar relacionado en los problemas emocionales (Salazar, Roldán, Garrido \& Parejo, 2014), ansiedad social (Aoki, Mearns \& Kurpius, 2017), agresividad (Thompson \& Berenbaum, 2011), adicciones (Tatari et al., 2020) y angustia (Vagos \& Pereira, 2018), que influyen en el desarrollo de psicopatologías y deterioro social del adolescente (Castaños, Reyes, Rivera \& Díaz, 2011).

La habilidad asertiva permite al individuo transmitir todo aquello que desea, sin ignorar o vulnerar los derechos de los demás (Moon, 2009; Postolati, 2017). Otros investigadores resaltan lo mencionado indicando que la conducta asertiva consiste en que las personas conozcan sus derechos personales y los defiendan (Castanyer, 1996; García \& Fábregas, 1988; Peneva \& Mavrodiev, 2013) de acuerdo al contexto social y las creencias básicas interpersonales (Vagos \& Pereira, 2010; 2016).

Asimismo, la conducta asertiva implica la expresión y aceptación eficaz de creencias, pensamientos, sentimientos u opiniones basado en el respeto de los demás (Castaños et al., 2011; Vagos \& Pereira, 2009). También, se considera como un factor guía en la interacción social debido a su importancia en la mejora de la comunicación interpersonal, $\mathrm{p}$ ues facilita concluir en acuerdos y compromisos ante opiniones opuestas (Naranjo, 2008). Contrariamente, la persona que carece de asertividad impone su opinión, sentimiento o pensamiento como "correcto" o "mejor" por encima de los demás sin respetar sus derechos (De Azevedo, 2016). El comportamiento asertivo es esencial para el desarrollo eficaz de relaciones interpersonales satisfactorias (Moon, 2009), y a que proporciona un mejor manejo y control de la conducta de manera asertiva como punto medio entre la agresividad y pasividad (Luna \& Ochoa, 2018).

Según Gambrill y Richey (1975) una persona asertiva presenta conductas verbales como: rechazar peticiones, responder a críticas, aceptar cumplidos, iniciar o mantener conversaciones. El comportamiento asertivo incluso puede evidenciarse mediante acciones no verbales reflejadas en el tono de la voz y el ritmo del habla adecuado a la situación social (Moon, 2009; Vagos \& Pereira, 2009), 
esto se vincula a un estado afectivo del comportamiento interpersonal carente de ansiedad o ira (Wolpe, 1973). La ausencia de los comportamientos asertivos puede generar reacciones cognitivas-emocionales negativas asociadas a la angustia e incomodidad subjetiva (Vagos \& Pereira, 2018).

Gambrill y Richey (1975) crearon el Inventario de Asertividad (IA), el cual fue aplicado a estudiantes universitarios de las ciudades de California y Washington compuesto por indicadores ante situaciones de interacción social a través de dos subescalas: La subescala de Grado de Incomodidad (GI) se basa en la incomodidad presentada frente una situación social específica. La subescala de Probabilidad de Respuesta (PR) denota que tan probable es que el evaluado realice dicha conducta. Ambas escalas presentan valores aceptables de confiabilidad de alfa de Cronbach, los cuales fueron GI de 0.87 y PR de .81. La subescala de GI a través del análisis factorial exploratorio (AFE) reportó 10 factores mediante rotación varimax, en el factor 1 (I9,I15,I17,I18), factor 2 (I26,I32,I35,I36,I38), factor 3 (I7,I8,I27,I28,I29), factor 4 (I5,I12,I14,I21,I24), factor 5 (I1,I3,I6,I11), factor $6(\mathrm{I} 4, \mathrm{I} 23, \mathrm{I} 25)$, factor 7 (I31, I34), factor 8 (I2,I16), factor 9 (I10,I19) y factor 10 (I13,I40).

En el panorama internacional encontramos a Carrasco, Clemente y Llavona (1989) que analizaron el instrumento con una muestra de 150 universitarios españoles de 19 a 21 años mediante el AFE, el cual determinó 12 factores para GI con una varianza total de $65.9 \%$ y 13 factores para PR con una varianza total de $65.7 \%$, que incluyen cargas cruzadas entre algunos ítems y evidencian coeficientes aceptables en ambos factores (GI; $\alpha=.91)$ y (PR; $\alpha=.87)$. En México encontramos a Guerra (1996) que realizó la adaptación del IA a través de 10 jueces expertos y una muestra de 1127 sujetos, el AFE demuestra que el inventario se dividió en 9 factores por cada subescala con una varianza total de $50 \%$ y $48 \%$ para ambas subescalas, cargas factoriales mayores a .40 , y valores altos de confiabilidad en las dos subescalas GI y PR con estimaciones de .92 y .88 , respectivamente.

Castaños y otros (2011) continúan con el estudio realizado por Guerra (1996) denominado "Estandarización del Inventario de Asertividad de Gambrill y Richey-II" versión mexicana. Las evidencias del AFE de cada subescala se componen en 8 factores para ambas subescalas de GI y PR, en esta versión se llega a eliminar 3 ítems (1, 4 y 22), se obtiene así un modelo de 37 factores con un coeficiente aceptable de alfa $(\alpha=.94)$ y por subescala (.91 y .89) respectivamente.

Otra investigación evaluó el IA en 759 universitarios chilenos (Navarro, Varas \& Maluenda, 2 015) mediante el AFE obteniendo 6 factores para cada subescala y fue eliminado el ítem 21 , este modelo de 39 reactivos presentó como resultados un coeficiente de alfa total de .95 y para cada subescala un alfa de .93 (GI) y .92 (PR). En México, Morales y Díaz (2018) continuaron con el estudio realizado inicialmente por Guerra (1996), en esta nueva investigación la medida 
del IA estuvo conformada por 5 factores (20 ítems) y buen índice de coeficiente de alfa total $(\alpha=.88)$.

En el contexto peruano fue adaptado por Sifuentes (2000), sin embargo, investigaciones realizadas posteriores mencionan que sus valoraciones de evidencias de validez y confiabilidad son cuestionables (Castaños et al., 2011; Navarro et al., 2015; Morales \& Díaz, 2018). Además, hasta la fecha no se han reportado estudios psicométricos de este instrumento en adolescentes peruanos . Por lo tanto, resulta pertinente la validación en contexto peruano dado la repercusión de la evaluación del comportamiento asertivo en los adolescentes que conduce a una buena comunicación y seguridad, esto refuerza la valía personal del adolescente que se siente respetado y satisfecho ante un buen desenvolvimiento asertivo en sus demandas escolares y sociales (Moon, 2009).

Las características asertivas son útiles en la aplicación de intervenciones psicológicas para reducir los niveles de malestar emocional como la depresión, ansiedad o el estrés en los adolescentes (Eslami, Rabiei, Afzali, Hamidizadeh \& Masoudi, 2016; Vagos \& Pereira, 2018; 2020).

Por ello, es necesario que todo instrumento validado, establezca la equivalencia de medición, para luego posteriormente evaluar las diferencias reales entre diferentes grupos (Byrne, 2008), esta característica psicométrica es esencial en la investigación psicológica de la asertividad, debido que hasta la fecha no se ha reportado evidencia de invarianza del instrumento .

En consecuencia, el objetivo de la investigación consistió en evaluar las propiedades psicométricas del Inventario de Asertividad de Gambrill y Richey (1975), para la población adolescente peruana con el fin de obtener evidencias de validez de la estructura interna, e invarianza de las dos subescalas que componen el inventario para su uso en contexto psicoeducativo cumpliendo con los estándares para su uso concreto como los proporcionados por The Standards for Educational and Psychological Testing (American Educational Research Association [AERA], American Psychological Association [APA] y el National Council on Measurement in Education [NCME], 2014).

\section{MÉTODO}

\section{Participantes}

La muestra de la presente investigación está constituida por 639 estudiantes peruanos (319 mujeres y 320 varones), con edades entre 15 a 20 años de edad ( $M E=15.84$, $D E=0.86)$ los cuales pertenecen al nivel secundario 4to (50.9\%) y 5 to $(49.1 \%)$ de cuatro instituciones educativas nacionales de la ciudad metropolitana de Lima. Se utilizó una calculadora en línea de tamaño a priori de Soper (2020) para calcular el tamaño de muestra necesario para el análisis factorial confirmatorio (AFC), dado 
el número de variables observadas y latentes en el modelo, el tamaño mínimo del efecto previsto de .30 , un nivel de probabilidad de .05 y potencia estadística de .95 (Ramos-Vera, 2020a).

\section{Instrumentos}

El Inventario de Asertividad está compuesto por dos sub escalas, los cuales las cuales indican el Grado de Incomodidad (GI) y la Probabilidad de Respuestas (PR), a través de 40 reactivos que plantean situaciones sociales diferentes.

La primera subescala está compuesta por 40 ítems de tipo Likert con 5 puntos y un escalamiento de respuesta de 1 al 5 , donde se responde qué tan incomodo se siente uno ante situaciones específicas: para nada (1), un poco (2), bastante (3), mucho (4) y muchísimo (5). En la segunda subescala se evaluaron los mismos 40 ítems de cinco puntos ( 1 al 5), donde se responde sobre cuan probable es realizar dicha situación social: siempre lo hago (1), habitualmente lo hago (2), lo hago a mitad de veces (3), casi nunca lo hago (4) y nunca lo hago (5).

\section{Procedimiento y Análisis estadísticos}

Se obtuvo la autorización de los autores del inventario, como también se requirió el permiso a los directores en las diferentes instituciones educativas mediante una solicitud formal dando a conocer el objetivo de la investigación. Estos se encargaron de informar los objetivos de la investigación tanto a los padres o tutores como a los estudiantes, con el fin de obtener el consentimiento y asentimiento de los mismos, así como dispone el artículo 24 del código de ética (Colegio de Psicólogos del Perú, 2017). El instrumento se respondió de manera voluntaria y anónima.

Para la va lidación del instrumento se realizó un proceso de traducción certificada de los ítems originales del instrumento (Muñiz, Elosua \& Hambleton, 2013). Posteriormente, s e valoró la adaptación lingüística a través de expertos definidos como profesionales que cuentan con experiencia en el tema (Escobar $\&$ Cuervo, 2008), lo que permitió modificar y reelaborar algunos términos con la finalidad de una mejor compresión de los ítems para la población adolescente. Se obtuvieron valores de $\mathrm{V}$ de Aiken aceptables, en donde se lograron valores mayores al mínimo establecido ( $\geq .70$ ]; Escurra, 1988). Se retiró 15 encuestas que omitieron varias respuestas quedando un total de 639 participantes.

Se verificó la existencia de los puntajes extremos a través de la asimetría y curtosis en el rango de -2 a 2 (Muthén \& Kaplan, 1985), convenientemente con matrices policóricas (Bandalos \& Finney, 2010; Ferrando y Anguiano-Carrasco, 2010).

Para brindar evidencias de validez basado en la estructura interna de los constructos, se procedió a realizar el análisis factorial confirmatorio a través del 
programa R Studio 3.0.1 bajo los siguientes criterios: el método de medias ponderadas de mínimos cuadrados y la estimación ajustada de la varianza (WLSMV) debido a que se aproximaron a una distribución multivariada y son datos ordinales (Beaducel \& Herzberg, 2006; Gana \& Broc, 2019); índices de ajuste como el SRMR $(\leq 0.05)$, RMSEA $(\leq 0.05)$, CFI $(\geq 0.95)$, y la proporción global $X^{2} / g l<3$ (Hu \& Bentler, 1999). También se evaluó la invarianza factorial del instrumento en función al sexo (Byrne, 2008). Finalmente, para verificar las evidencias de la confiabilidad de los instrumentos se analizó a través del método de consistencia interna con los coeficientes $\alpha$ y $\omega \geq .70$ (Hair, Black, Babin \& Anderson, 2014).

Se evaluó la comparación según sexo mediante la prueba t bayesiana (Ramos-Vera, 2021a; 2021b) para seleccionar aquellas diferencias con evidencia concluyente (factor Bayes [FB] > 10), está medida bayesiana permite confirmar la validez de la hipótesis alterna de diferencia más allá del rechazo de la hipótesis nula, se consideró una distribución de Cauchy (medida en una escala de "r") centrada en $0 \mathrm{y}$ un valor intercuartil de .707 .

\section{RESULTADOS}

A partir de la traducción y la revisión lingüística del IA de Gambrill y Richey (1975) se decidió modificar la redacción de 32 ítems según el criterio de jueces (ver tabla 1) y reelaborar 7 reactivos que se utilizan en ambas subescalas para la evaluación de su uso en el contexto adolescente (ver tabla 2).

Tabla 1

Reactivos que cambiaron de redacción

\begin{tabular}{|c|c|}
\hline Ítems originales & Ítems adaptados \\
\hline 1.Decir que no cuando te piden prestado el automóvil & 1. Te niegas a prestar tu celular cuando te piden. \\
\hline 2.Dar un cumplido a un amigo & 2. Felicitas a un amigo. \\
\hline 3.Pedir un favor a alguien & 3.Pides un favor a alguien \\
\hline 4.Resistir a las insistencias de un vendedor & 4.Resistes la presión de los vendedores (de ofertas) \\
\hline 5.Pedir disculpas cuando has cometido un error & 5.Pides disculpas cuando cometes una falta \\
\hline 10.Admitir tu ignorancia en algunos temas & 10. Admites cuando desconoces un tema. \\
\hline 11.Decir que no cuando alguien te pide dinero prestado & 11.Te niegas a prestar dinero cuando te piden \\
\hline 12.Hacer preguntas personales & 12.Haces preguntas de carácter personal \\
\hline 13.Parar la conversación con un amigo muy hablador & 13. Cortas la palabra a un amigo que habla demasiado. \\
\hline 14.Pedir críticas constructivas & 14. Pides críticas constructivas. \\
\hline 15.Iniciar una conversación con un extraño & 15. Con una persona extraña inicio una conversación. \\
\hline $\begin{array}{l}\text { 16.Dar un cumplido a una persona con quien tienes una } \\
\text { relación íntima o en quien estás interesado/a }\end{array}$ & $\begin{array}{l}\text { 16. Haces un cumplido a la persona por quien sientes } \\
\text { interés sentimental o amoroso }\end{array}$ \\
\hline 17.Concertar una cita o reunión con una persona & 17. Pides tener un encuentro o cita con una persona. \\
\hline $\begin{array}{l}\text { 18.Tu pedido inicial para una reunión con alguien es } \\
\text { rechazado y vuelves a pedirle verlo/a más adelante }\end{array}$ & $\begin{array}{l}\text { 18. Si tu pedido para una cita es rechazado, pides otra } \\
\text { para otro momento. }\end{array}$ \\
\hline
\end{tabular}


Tabla 1. Continuación

\begin{tabular}{|c|c|}
\hline Ítems originales & Ítems adaptados \\
\hline 20.Postular a un empleo & 20. Buscas proactivamente un empleo. \\
\hline 21.Preguntar si has ofendido a alguien & 21. Si crees que le has ofendido a alguien le preguntas. \\
\hline 22.Decirle a alguien que te gusta & 22.Le dices a alguien que te agrada \\
\hline $\begin{array}{l}\text { 24.Discutir abiertamente con otra persona cuando él o } \\
\text { ella ha criticado tu comportamiento }\end{array}$ & $\begin{array}{l}\text { 24.Discutes abiertamente con una persona sobre su } \\
\text { crítica a tu comportamiento }\end{array}$ \\
\hline $\begin{array}{l}\text { 25.Devolver un artículo defectuoso, como en una tienda } \\
\text { o restaurante }\end{array}$ & $\begin{array}{l}\text { 25. Devuelves el producto equivocado, por ejemplo: a } \\
\text { una tienda o restaurante. }\end{array}$ \\
\hline $\begin{array}{l}\text { 26.Expresar una opinión diferente a la de la persona con } \\
\text { quien estás hablando }\end{array}$ & $\begin{array}{l}\text { 26. Expresar una opinión diferente cuando hablas con } \\
\text { otra persona. }\end{array}$ \\
\hline $\begin{array}{l}\text { 28.Poder decirle a alguien que sientes que ha hecho algo } \\
\text { que no es justo contigo }\end{array}$ & $\begin{array}{l}\text { 28. Le dices a una persona que sientes que él o ella han } \\
\text { hecho algo que es injusto para ti. }\end{array}$ \\
\hline 29.Aceptar una cita & 29. Aceptar una cita o invitación. \\
\hline 30.Contarle a alguien buenas nuevas sobre ti mismo & $\begin{array}{l}\text { 30. Le cuentas a alguien las buenas noticias acerca de } \\
\text { ti mismo. }\end{array}$ \\
\hline 31.Resistir a la presión de tomar alcohol & 31. Resistes la presión de tomar bebidas alcohólicas. \\
\hline $\begin{array}{l}\text { 32.Rechazar las exigencias injustas de una persona que } \\
\text { es importante para ti }\end{array}$ & $\begin{array}{l}\text { 32. Rechazas un pedido injusto de una persona impor- } \\
\text { tante. }\end{array}$ \\
\hline 33.Renunciar a un trabajo & 33. Te retiras de un trabajo que no te conviene. \\
\hline 34.Resistir a la presión de usar drogas & 34. Resistes presión para consumir drogas. \\
\hline $\begin{array}{l}\text { 35.Discutir abiertamente con alguien que ha criticado } \\
\text { tu trabajo }\end{array}$ & $\begin{array}{l}\text { 35. Discutes abiertamente con alguien, acerca de su } \\
\text { crítica a tu trabajo. }\end{array}$ \\
\hline 36.Pedir que te devuelvan un artículo prestado & 36. Pides que te devuelvan los artículos que prestaste. \\
\hline 37.Aceptar un cumplido & 37. Aceptas halagos o felicitaciones. \\
\hline $\begin{array}{l}\text { 38. Continuar conversando con alguien que no concuer- } \\
\text { da contigo en un tema }\end{array}$ & $\begin{array}{l}\text { 38.Continúas conversando con alguien que no está de } \\
\text { acuerdo contigo }\end{array}$ \\
\hline $\begin{array}{l}\text { 40.Pedirle a una persona que te está molestando en } \\
\text { público que pare }\end{array}$ & $\begin{array}{l}\text { 40. Pides a la persona que te está molestando pública- } \\
\text { mente que se detenga. }\end{array}$ \\
\hline
\end{tabular}

Nota: ítems en los cuales se ha modificaron de alguna forma en su redacción.

\section{Tabla 2}

Reactivos que fueron reelaborados

\begin{tabular}{|c|c|}
\hline Ítems originales & Ítems adaptados \\
\hline 6. Rechazar una invitación a una reunión o cita & $\begin{array}{l}\text { 6. Si te desagrada dices no a una asistencia de una } \\
\text { reunión o cita. }\end{array}$ \\
\hline $\begin{array}{l}\text { 7.Admitir que tienes miedo y pedir que te muestren } \\
\text { consideración }\end{array}$ & 7. Admites sentir miedo y pides comprensión. \\
\hline $\begin{array}{l}\text { 8.Decirle a una persona con quien tienes una relación } \\
\text { íntima que algo que ha dicho o hecho te molesta }\end{array}$ & $\begin{array}{l}\text { 8. Le aclaras a un familiar o amigo cuando dicen algo } \\
\text { que te molesta. }\end{array}$ \\
\hline 9.Pedir un aumento de sueldo & $\begin{array}{l}\text { 9. Pides reconocimiento cuando tengo un logro } \\
\text { personal. }\end{array}$ \\
\hline $\begin{array}{l}\text { 19.Admitir que tienes dudas respecto a un tema de la } \\
\text { conversación y pedir que lo aclaren }\end{array}$ & $\begin{array}{l}\text { 19. Pides que te aclaren cuando te has confundido sobre } \\
\text { un punto en discusión. }\end{array}$ \\
\hline $\begin{array}{l}\text { 23. Reclamar un servicio esperado cuando este no llega. } \\
\text { Por ejemplo, en un restaurante }\end{array}$ & $\begin{array}{l}\text { 23.Pides que te atiendan, cuando los encargados no lo } \\
\text { hacen, por ejemplo: en un restaurante }\end{array}$ \\
\hline $\begin{array}{l}\text { 27.Resistir propuestas sexuales cuando no estás } \\
\text { interesado }\end{array}$ & $\begin{array}{l}\text { 27. Rechazas insinuaciones románticas cuando no estas } \\
\text { interesado. }\end{array}$ \\
\hline
\end{tabular}

Nota: reactivos que llegaron a ser reelaborados más no diferidos de la versión original. 


\section{Descripción de puntajes extremos}

De acuerdo a los resultados obtenidos del análisis descriptivo para el instrumento, presentaron valores de asimetría y curtosis dentro del rango esperado -2 a 2 (Muthén \& Kaplan, 1985; Bandalos \& Finney, 2010), se procedió el análisis considerando el método robusto de estimación (WLSMV) para las estimaciones de la validez basada en la estructura interna (ver tabla 3).

Tabla 3

Datos Preliminares de la subescala de grado de incomodidad e indice de respuesta

\begin{tabular}{|c|c|c|c|c|c|c|c|c|c|}
\hline Ítem & M & DE & g1 & g2 & Ítem & M & DE & g1 & g2 \\
\hline I1 & 2.21 & 1.14 & 0.04 & 0.30 & $\mathrm{P} 1$ & 3.00 & 1.27 & 0.05 & -1.01 \\
\hline I2 & 2.33 & 1.23 & 0.05 & -0.66 & P2 & 2.41 & 1.09 & 0.04 & -0.52 \\
\hline I3 & 2.41 & 1.05 & 0.04 & -0.24 & P3 & 2.64 & 1.06 & 0.04 & -0.57 \\
\hline I4 & 2.66 & 1.30 & 0.05 & -0.99 & P4 & 2.67 & 1.26 & 0.05 & -0.98 \\
\hline I5 & 2.77 & 1.35 & 0.05 & -1.14 & P5 & 2.03 & 1.12 & 0.04 & 0.13 \\
\hline I6 & 2.42 & 1.15 & 0.05 & -0.29 & P6 & 2.65 & 1.18 & 0.05 & -0.85 \\
\hline I7 & 2.41 & 1.22 & 0.05 & -0.50 & P7 & 2.96 & 1.22 & 0.05 & -0.99 \\
\hline I8 & 2.59 & 1.22 & 0.05 & -0.77 & P8 & 2.56 & 1.19 & 0.05 & -0.72 \\
\hline I9 & 2.17 & 1.19 & 0.05 & -0.08 & P9 & 3.22 & 1.28 & 0.05 & -1.06 \\
\hline $\mathrm{I} 10$ & 2.44 & 1.15 & 0.05 & -0.56 & P10 & 2.51 & 1.11 & 0.04 & -0.60 \\
\hline I11 & 2.27 & 1.11 & 0.04 & -0.06 & P11 & 3.05 & 1.12 & 0.04 & -0.77 \\
\hline I12 & 2.29 & 1.13 & 0.04 & -0.22 & P12 & 3.00 & 1.17 & 0.05 & -0.85 \\
\hline I13 & 2.25 & 1.17 & 0.05 & -0.31 & P13 & 3.34 & 1.20 & 0.05 & -0.79 \\
\hline I14 & 2.20 & 1.11 & 0.04 & 0.01 & P14 & 2.98 & 1.22 & 0.05 & -0.92 \\
\hline I15 & 2.36 & 1.22 & 0.05 & -0.42 & P15 & 3.25 & 1.23 & 0.05 & -0.90 \\
\hline I16 & 2.44 & 1.36 & 0.05 & -0.80 & P16 & 2.94 & 1.35 & 0.05 & -1.16 \\
\hline I17 & 2.14 & 1.21 & 0.05 & -0.11 & P17 & 3.52 & 1.25 & 0.05 & -0.97 \\
\hline I18 & 2.12 & 1.28 & 0.05 & -0.31 & P18 & 3.70 & 1.34 & 0.05 & -0.95 \\
\hline I19 & 2.44 & 1.14 & 0.04 & -0.50 & P19 & 2.53 & 1.12 & 0.04 & -0.52 \\
\hline I 20 & 2.17 & 1.18 & 0.05 & -0.11 & P20 & 3.15 & 1.33 & 0.05 & -1.17 \\
\hline I 21 & 2.56 & 1.25 & 0.05 & -0.84 & P21 & 2.64 & 1.23 & 0.05 & -0.80 \\
\hline I22 & 2.47 & 1.30 & 0.05 & -0.88 & P22 & 2.68 & 1.29 & 0.05 & -0.96 \\
\hline I 23 & 2.62 & 1.29 & 0.05 & -0.91 & P23 & 2.73 & 1.21 & 0.05 & -0.79 \\
\hline I 24 & 2.35 & 1.13 & 0.04 & -0.17 & P24 & 3.04 & 1.21 & 0.05 & -0.91 \\
\hline $\mathrm{I} 25$ & 2.47 & 1.27 & 0.05 & -0.85 & $\mathrm{P} 25$ & 2.95 & 1.28 & 0.05 & -1.01 \\
\hline I26 & 2.39 & 1.17 & 0.05 & -0.48 & P26 & 2.74 & 1.16 & 0.05 & -0.62 \\
\hline $\mathrm{I} 27$ & 2.64 & 1.37 & 0.05 & -1.08 & P27 & 2.71 & 1.32 & 0.05 & -1.05 \\
\hline I 28 & 2.56 & 1.28 & 0.05 & -0.90 & P28 & 2.74 & 1.23 & 0.05 & -0.82 \\
\hline I 29 & 2.38 & 1.23 & 0.05 & -0.53 & & & & & \\
\hline I30 & 2.50 & 1.29 & 0.05 & -0.80 & & & & & \\
\hline I31 & 2.63 & 1.46 & 0.06 & -1.19 & & & & & \\
\hline I32 & 2.51 & 1.21 & 0.05 & -0.67 & & & & & \\
\hline I33 & 2.57 & 1.34 & 0.05 & -1.05 & & & & & \\
\hline I34 & 2.73 & 1.60 & 0.06 & -1.49 & & & & & \\
\hline I35 & 2.42 & 1.19 & 0.05 & -0.66 & & & & & \\
\hline I36 & 2.68 & 1.33 & 0.05 & -1.02 & & & & & \\
\hline I37 & 2.61 & 1.36 & 0.05 & -1.02 & & & & & \\
\hline I38 & 2.41 & 1.16 & 0.05 & -0.51 & & & & & \\
\hline I39 & 2.60 & 1.29 & 0.05 & -0.93 & & & & & \\
\hline I40 & 2.77 & 1.40 & 0.06 & -1.20 & & & & & \\
\hline
\end{tabular}

Nota: $M$ : media, $D E$ : desviación estándar, $g 1$ : asimetría, $g 2$ : curtosis

\section{Análisis confirmatorio de la subescala de grado de incomodidad}

El AFC es el procedimiento apropiado para ajustar estructuras factoriales, en este caso, la escala de asertividad de Gambrill y Richey confirma su estructura interna 
demostrando cuatro modelos, en la subescala de GI contamos con el modelo de 40 ítems está agrupado de 5 factores y demuestran cargas factoriales menores .30 por debajo de los parámetros requeridos (mayores a .40) (Brown, 2006), es por ello que se restructura obteniendo el modelo de 38 ítems, el cual se retira dos ítems $(4$ y 18), en los modelos de segundo orden y unifactorial se mantienen los 38 ítems. En la sub escala de PR contamos con el modelo de 40 ítems el cual mostraba cargas factoriales por debajo de los criterios psicométricos referidos (Brown, 2006), y se realiza la restructuración obteniendo el modelo de 29 ítems (ítems eliminados: 1, $3,4,5,10,11,13,15,17,18$ y 20$)$.

En la tabla 4, podemos verificar los indicadores de ajuste de bondad de ajuste para cada una de las subescalas. En el primer modelo de 40 ítems de la subescala de grado de incomodidad de 5 factores, presentan indicadores aceptables $\left(X^{2} / \mathrm{gl}=\right.$ $1.83, \mathrm{CFI}=.98, \mathrm{TLI}=.98, \mathrm{SRMR}=.05$, RMSEA $[\mathrm{IC}-90 \%]=.036[.033, .039])$, al igual que el resto de modelos con valores similares, en el modelo de 38 ítems también evidencia valores adecuados $\left(X^{2} / \mathrm{gl}=1.61, \mathrm{CFI}=.99, \mathrm{TLI}=.99, \mathrm{SRMR}=.05\right.$, RMSEA [IC-90\%] = .031 [.027, .034]), contando así también con valores idénticos en el modelo de segundo orden $\left(X^{2} / \mathrm{gl}=1.61, \mathrm{CFI}=.99, \mathrm{TLI}=.99, \mathrm{SRMR}=.05, \mathrm{RM}-\right.$ SEA $[$ IC-90\%] $=.031[.027, .034])$.

\section{Tabla 4}

Indicadores de ajuste del modelo estructural confirmatorio de la sub escala de grado de incomodidad del Inventario de Asertividad de Gambrill y Richey e Invarianza

\begin{tabular}{lcccccccc}
\hline Modelo & $\mathbf{X}^{\mathbf{2}}$ & $\mathbf{g l}$ & $\mathbf{X}^{2} / \mathbf{g l}$ & $\mathbf{p}$ & $\mathbf{C F I}$ & TLI & SRMR & RMSEA \\
\hline 40 ítems & 1336.11 & 730 & 1.83 & $<.001$ & 0.98 & 0.98 & 0.05 & 0.036 \\
38 ítems & 1054.57 & 655 & 1.61 & $<.001$ & 0.99 & 0.99 & 0.05 & 0.031 \\
2do orden & 1060.8 & 660 & 1.61 & $<.001$ & 0.99 & 0.99 & 0.05 & 0.031 \\
\hline \multicolumn{1}{l}{ Invarianza subescala de grado de incomodidad } & & & & & \\
\hline Modelo & $\mathbf{X}^{2}$ & $\mathbf{g l}$ & $\Delta \mathbf{X}^{2}$ & $\Delta$ gl & $\mathbf{C F I}$ & $\mathbf{R M S E A}$ & $\Delta \mathbf{C F I}$ & $\Delta \mathbf{R M S E A}$ \\
\hline Modelo 1 & 2686.5 & 1310 & - & - & 0.950 & 0.060 & - & - \\
Modelo 2 & 2750.7 & 1343 & 64.15 & 33 & 0.950 & 0.059 & 0.000 & 0.001 \\
Modelo 3 & 2822.7 & 1376 & 72.01 & 33 & 0.951 & 0.058 & 0.001 & 0.001 \\
Modelo 4 & 2897.1 & 1414 & 74.39 & 38 & 0.952 & 0.059 & 0.001 & 0.001 \\
Modelo 5 & 2907.4 & 1419 & 10.32 & 5 & 0.952 & 0.058 & 0.000 & 0.001 \\
\hline
\end{tabular}

Nota.: Chi cuadrado: $x 2$, gl: grado de libertad, p: significancia de ajuste, CFI: índice de ajuste comparativo, TLI: índice de Tucker Lewis, SRMR: Residual estandarizada cuadrática media, RMSEA: Error cuadrática media de aproximación. X2: Chi Cuadrado, $\Delta \mathrm{X} 2=$ Chi Cuadrado delta, $\Delta \mathrm{gl}$ : grados de libertad delta. $\Delta \mathrm{CFI}$ : Índice de ajuste Comparativo delta, $\Delta$ RMSEA: Error Cuadrático Medio de Aproximación delta.

En la tabla 5, encontramos los indicadores de ajuste de la subescala de PR, tales modelos propuestos también fueron aceptables según la literatura psicométrica, el primer modelo de 40 ítems presenta valores adecuados $\left(X^{2} / \mathrm{gl}=2.27, \mathrm{CFI}=\right.$ $.91, \mathrm{TLI}=.90, \mathrm{SRMR}=.06, \mathrm{RMSEA}[\mathrm{IC}-90 \%]=.045[.042, .047])$. El modelo de 
29 ítems presenta valores óptimos $\left(X^{2} / \mathrm{gl}=1.75, \mathrm{CFI}=.96, \mathrm{TLI}=.96, \mathrm{SRMR}=.05\right.$, RMSEA [IC-90\%] =.034 [.030, .039]), contando así mismo con un modelo de segundo orden con valores similares $\left(X^{2} / \mathrm{gl}=1.78, \mathrm{CFI}=.96, \mathrm{TLI}=.96, \mathrm{SRMR}=.05\right.$, RMSEA $[\mathrm{IC}-90 \%]=.035[.031, .039])$.

\section{Tabla 5}

Indicadores de ajuste del modelo estructural confirmatorio de la sub escala de probabilidad de respuesta del Inventario de Asertividad de Gambrill y Richey e Invarianza

\begin{tabular}{lcccccccc}
\hline Ítems & $\mathbf{X}^{\mathbf{2}}$ & $\mathbf{g l}$ & $\mathbf{X}^{2} / \mathbf{g l}$ & $\mathbf{P}$ & $\mathbf{C F I}$ & $\mathbf{T L I}$ & SRMR & RMSEA \\
\hline 40 ítems & 1654.67 & 730 & 2.27 & $<.001$ & 0.91 & 0.90 & 0.06 & 0.045 \\
29 ítems & 643.98 & 367 & 1.75 & $<.001$ & 0.96 & 0.96 & 0.05 & 0.034 \\
2do orden & 660.97 & 372 & 1.78 & $<.001$ & 0.96 & 0.96 & 0.05 & 0.035 \\
\hline Invarianza & $\mathbf{X}^{\mathbf{2}}$ & $\mathbf{g l}$ & $\Delta \mathbf{X}^{\mathbf{2}}$ & $\Delta \mathbf{g l}$ & $\mathbf{C F I}$ & $\mathbf{R M S E A}$ & $\Delta$ CFI & $\Delta$ RMSEA \\
\hline M1 & 1386.6 & 734 & - & - & 0.960 & 0.055 & - & \\
M2 & 1410.9 & 758 & 24.31 & 24 & 0.965 & 0.060 & 0.005 & 0.005 \\
M3 & 1488.5 & 782 & 77.61 & 24 & 0.970 & 0.055 & 0.005 & 0.005 \\
M4 & 2897.1 & 1414 & 74.39 & 38 & 0.965 & 0.059 & 0.005 & 0.004 \\
M5 & 2907.4 & 1419 & 10.32 & 5 & 0.966 & 0.058 & 0.001 & 0.001 \\
\hline
\end{tabular}

Nota: Chi cuadrado, gl: grado de libertad, p: significancia de ajuste, CFI: índice de ajuste comparativo, TLI: índice de Tucker Lewis, SRMR: Residual estandarizada cuadrática media, RMSEA: Error cuadrática media de aproximación. X2: Chi Cuadrado, $\Delta \mathrm{X} 2=$ Chi Cuadrado delta, $\Delta \mathrm{gl}$ : grados de libertad delta. $\Delta \mathrm{CFI}$ : Índice de ajuste Comparativo delta, $\triangle$ RMSEA: Error Cuadrático Medio de Aproximación delta.

Se determinó la equivalencia factorial para ambas subescalas del instrumento a través de M1, M2, M3, M4 y M5, siendo así que la subescala GI presentó buenos valores de invarianza configuracional (M1), CFI $=.95$ y $\triangle \mathrm{RMSEA}=.06$, procediendo así posteriormente al modelo de invarianza métrica (M2), hallando índices adecuados de ajuste: $\triangle \mathrm{CFI}=.000 \mathrm{y} \triangle \mathrm{RMSEA}=.000$, presentan valor similar a M1, dentro de los parámetros, $\Delta$ CFI $\leq .01$ y $\triangle$ RMSEA $\leq .01$ (Chen, 2007; Cheung \& Rensvold, 2002), indicando la conclusión de la equivalencia de las cargas factoriales, siendo así posible comparar las varianzas. Posteriormente, s e evaluó la equivalencia de los umbrales o invarianza fuerte (M3) cumpliendo también con los estándares $(\triangle \mathrm{RMSEA}<.01$ y $\triangle \mathrm{CFI}<.01$, Cheung \& Rensvold, 2002), $\Delta \mathrm{CFI}=.000$ y $\triangle \mathrm{RMSEA}=.000$. $\mathrm{D}$ e igual manera se aceptó la invarianza de los interceptos (M3) cumpliendo los estándares de invarianza referidos. Luego se prosiguió con la examinación de la varianza estricta (M4) agregando la invarianza de residuos, evidenciando la diferencia mínima con el modelo anterior confirmando la invarianza residual. Se estimó las medias latentes después de obtener las invarianzas anteriores (Dimitrov, 2010; Milfont \& Fischer, 2010). De igual manera se cumplió con todos los parámetros de las medidas consecutivas de la invarianza factorial (configuracional, métrica, fuerte, residuos y medias latentes) de la subescala PR demostrando también que es invariante. 


\section{Análisis de Confiabilidad}

La confiabilidad de la sub escala de grado de incomodidad y sus factores del Inventario de Asertividad de Gambrill y Richey se realizó por el método de consistencia interna mediante el coeficiente alfa, la subescala GI obtuvo un valor de .93, por lo que se considera aceptable (Nunnally \& Berstein, 1995). Asimismo, se determinó los coeficientes de consistencia interna por factores con estimaciones entre .72 a .89. Igualmente se tuvo en cuenta el coeficiente omega para las evidencias de confiabilidad según las subescalas (tabla 6).

\section{Tabla 6}

Confiabilidad de la sub escala de grado de incomodidad y sus factores del Inventario de Asertividad de Gambrill y Richey

\begin{tabular}{lccc}
\hline Variable & $\boldsymbol{\alpha}$ & $\boldsymbol{\omega}$ & $\mathbf{N}$ \\
\hline Sub escala - Grado de incomodidad & 0.92 & 0.93 & 38 \\
Negación & 0.79 & 0.79 & 7 \\
Solicitudes & 0.72 & 0.72 & 6 \\
Situaciones agradables/interacción & 0.81 & 0.81 & 10 \\
Aceptar deficiencias personales & 0.79 & 0.79 & 6 \\
Situaciones incomodas & 0.88 & 0.89 & 9 \\
\hline
\end{tabular}

Nota. $\alpha$ : alfa, $\omega$ : omega, IA-VP: versión peruana del Inventario de Asertividad, N: número de indicadores.

Se calculó la confiabilidad por consistencia interna mediante el coeficiente alfa de la sub escala de probabilidad de respuesta del Inventario de Asertividad de Gambrill y Richey, el cual fue de .86. Asimismo, se determinó los valores de confiabilidad para cada dimensión encontrándose valores de .70 a .78. Por otro lado, también se empleó el coeficiente omega para ver la confiabilidad por consistencia interna, el cual arrojó mejores resultados para la sub escala total y cada uno de sus factores (ver tabla 7).

Tabla 7

Confiabilidad de la sub escala (PR) y sus factores del Inventario de Asertividad de Gambrill y Richey

\begin{tabular}{lccc}
\hline Variable & $\boldsymbol{\alpha}$ & $\boldsymbol{\omega}$ & $\mathbf{N}$ \\
\hline Sub escala - Probabilidad de respuesta & 0.86 & 0.86 & 29 \\
Negación & 0.78 & 0.78 & 5 \\
Solicitudes & 0.75 & 0.76 & 2 \\
Situaciones agradables/interacción & 0.70 & 0.71 & 9 \\
Aceptar deficiencias personales & 0.76 & 0.77 & 5 \\
Situaciones incomodas & 0.73 & 0.74 & 8 \\
\hline
\end{tabular}

Nota. $\alpha$ : alfa, $\omega$ : omega, IA-VP: versión peruana del inventario de asertividad, $N$ : número de ítems 


\section{Análisis diferencial según sexo}

Se compararon mediante la prueba $\mathrm{T}$ de student bayesiano en función del sexo con respecto a las subescalas y sus dimensiones respectivas, los hallazgos indicaron diferencias con evidencia débil según sexo en las puntuaciones de los factores y las subescalas (FB $\leq 3$ ), esto no permite confirmar la validez de hipótesis de diferencia de grupos. Dado que, en contraposición al test de hipótesis, que tiende a dicotomizar los hallazgos, vamos a cuantificar la probabilidad de las evidencias a favor de las hipótesis (nula y alternativa).

\section{DISCUSIÓN}

En la actualidad la política educativa y pedagógica han establecido reglas, registros y medidas de comportamientos y sentimientos, así como estilos establecidos de interacción de los adolescentes con las normas sin considerar los pensamientos, voluntades, anhelos y la cultura, lo cual reduce mucho la libertad y responsabilidad de los estudiantes para interactuar socialmente (Chávez-Kanashiro \& Garrido-Arévalo, 2015; Cueva, Hernández \& Regalado, 2021). Además, muchos profesores pueden ser estrictos y autoritarios lo que puede generar diferencias con los estudiantes, quienes deben ser asertivos para gestionar tales diferencias con los docentes, o con los compañeros y familiares.

La presente investigación tuvo como objetivo principal evaluar las propiedades psicométricas del Inventario de Asertividad de Gambrill y Richey (1975), dado que, dicha variable favorece a las capacidades para desarrollar límites directa y oportunamente con el propósito de respetar a su entorno social y personal. Esto es importante en el desarrollo de nuevas capacidades y habilidades sociales en los adolescentes (Gaeta \& Galvanovskis, 2009),

En cuanto a las evidencias de confiabilidad derivadas de la aplicación de los ítems, el estudio previo solo incluyó el coeficiente alfa, sin embargo, para esta investigación se consideró añadir el coeficiente omega, porque presenta dos fortalezas: permite el manejo de la suma de las variables estandarizadas que genera estabilidad en el cálculo de la fiabilidad y no presenta sesgo por la cantidad de reactivos (Deng \& Chang, 2017), los valores de confiabilidad de las subescalas y la puntuación total refieren valores aceptables.

Se realizó el análisis estructural en ambas subescalas, presentando adecuados índices de ajuste a los modelos de medición de cinco factores de 38 ítems en la subescala (GI) y el modelo de cinco factores de 28 ítems en la subescala PR, esto es similar al estudio de Navarro et al. (2016) en universitarios chilenos, siendo probable que los adolescentes peruanos tengan la capacidad de distinguir estos cinco dominios afines a la asertividad. 
La adaptación del instrumento al ámbito psicoeducativo permite evaluar mediante las dos subescalas, la primera vinculada a la incomodidad de determinadas conductas sociales (baja asertividad) orientada a la ansiedad social (Vagos \& Pereira, 2020), mientras la otra subescala evaluó la expresión y afinidad a tales situaciones sociales (alta asertividad) que permite medir las habilidades asertivas de los adolescentes según el modelo propuesto por Gambrill y Richey (1975). Dicha característica es esencial en los estudiantes, quienes deben ser capaces de establecer relaciones sociales positivas que favorezcan su desarrollo y ajuste psicológico vinculado a una mayor autorregulación emocional y conductual (Farley \& Kim-Spoon, 2014), que afiance un mayor rendimiento académico (Laursen \& Mooney, 2008), y un comportamiento idóneo para enfrentar las demandas sociales y escolares (Plenty, Östberg, Almquist, Augustine \& Modin, 2014).

Respecto al análisis de invarianza, como precedente a la investigación psicológica peruana se comprobó su precisión, en la búsqueda de estimación de un mismo modelo en ambas subescalas del instrumento entre varones y mujeres (Byrne, 2008), que refiere el carácter invariante respecto al género (Millsap \& OliveraAguilar, 2012) correspondiente a lo recomendado por The Standards for Educational and Psychological Testing (AERA, APA \& NCME, 2014). Dado que hasta la fecha no se precisan estudios instrumentales previos de la capacidad asertiva en adolescentes de habla hispana. E studios nacionales recientes también señalan que las habilidades psicológicas pueden ser invariantes según sexo en adolescentes (Ramos-Vera, Ramírez, Rojas, Serpa \& García, 2021; Ramos-Vera, Serpa \& Lemos, 2020). Se debe resaltar que la equivalencia de medición considera las diferencias del constructo evaluado y no según las características de la muestra específica (Byrne, 2008). Es decir, que después de obtener los resultados de invarianza de medición e s posible proponer hipótesis comparativas generalizables según los datos respectivos.

Se consideró la prueba t bayesiana para evaluar las diferencias según sexo, los hallazgos solo señalan una evidencia débil, que indica que no es posible confirmar la verosimilitud de las hipótesis a favor de diferencia de las puntuaciones entre varones y mujeres. Aunque otros estudios reportaron diferencias de menores capacidades asertivas a favor de los varones (Albornoz, Navarro \& Varas, 2017; Cerquera, 2020; Sarmiento, González, Hermida, Celis \& Nieves, 2021). Tales investigaciones fueron basadas en las diferencias de hipótesis de significancia y no consideraron el criterio bayesiano para el contraste de las hipótesis, por tanto, los hallazgos bayesianos reportados son considerados como un aporte metodológico para la investigación psicológica. La medida refiere adecuadas evidencias de validez e invariabilidad para la evaluación en futuras intervenciones psicoterapéuticas en hombres y mujeres sin posibles sesgos de medición (Milovanovic, 2021; Sodikin, Keliat \& Wardani, 2021). Además, es posible que 
la capacidad asertiva sea aprendida por los estudiantes a través de programas psicoeducativos (p. ej., Parray, Kumar, David, Khare \& Maurya, 2020; Vagos \& Pereira, 2016) para la reducción de comportamientos interpersonales vinculados a la agresión y sumisión, esencial en las interacciones positivas del aula (Vagos \& Pereira, 2010).

El estudio presenta algunas limitaciones. Primeramente, debido a razones de accesibilidad, solo se incluyeron los escolares de instituciones educativas nacionales, mientras que aquellos de las privadas no fueron considerados. De esta manera, la generalización de los resultados ha podido verse afectada debido a las características de los participantes. Es necesario realizar más estudios psicométricos en diversas poblaciones estudiantiles según grupos de edad, nivel socioeconómico, grupo étnico, etc., para fortalecer la comparabilidad y la equivalencia del constructo en adolescentes.

Es importante el uso de otros enfoques como el factor Bayes (Ramos-Vera, 2020b) para evaluar la fuerza probatoria en las evaluaciones comparativas y en las relaciones estadísticas que refuercen las evidencias de validez convergente en futuros estudios de la presente revista, dado que permite precisar la probabilidad de verosimilitud de las hipótesis estadísticas (p. Ej., Núñez. Ramos-Vera, Serpa \& Ogundokun, 2021). Otro método útil es el análisis de red de relaciones estadísticas (Ramos-Vera, 2021c; Ramos-Vera \& Serpa, 2021) que requiere mayor difusión en la investigación de medición psicológica sudamericana (p ej., Ramos-Vera, Dávila. Castañeda, Ramírez \& Serpa, 2021)

La medición del instrumento se realizó transversalmente, por lo que estudios longitudinales brindarían mayor evidencia de validez predictiva y se recomienda la aplicación de la confiabilidad test-retest para futuras investigaciones.

En conclusión, el instrumento propuesto muestra adecuadas propiedades psicométricas en cuanto a su validez basado al contenido, la estructura interna, invarianza y las estimaciones de confiabilidad para su uso en el contexto peruano. Además, la prueba se puede utilizar a nivel nacional, dado su facilidad y rapidez en la administración.

\section{Fuentes de financiamiento}

La investigación fue autofinanciada por los propios autores.

\section{Conflicto de intereses}

Los autores declaran no tener ningún conflicto de interés económico, institucional, laboral o personal al realizar el manuscrito. 


\section{REFERENCIAS}

Albornoz, J. M., Saldaña, G. N., \& Contreras, M. V. (2017). Asertividad: Diferencias de sexo en estudiantes universitarios chilenos medidas a través del Inventario de Gambrill y Richey. REXE: Revista de estudios y experiencias en educación, 16(31), 5568. Disponible en: https://dialnet.unirioja.es/descarga/articulo/6067472.pdf

American Educational Research Association, American Psychological Association, \& National Council on Measurement in Education (2014). Standards for Educational and Psychological Testing. Washington, DC: American Educational Research Association. Recuperado de: https://www.apa.org/science/programs/testing/standards.

Aoki, S., Mearns, J., \& Kurpius, S. (2017). Social anxiety and assertiveness: The role of self-beliefs in asian americans and european americans. Journal of Mental Health Counseling, 39(3), 263-274. DOI: http://dx.doi.org/10.17744/mehc.39.3.06

Bandalos, D., \& Finney, S. (2010) Reviewer's guide to quantitative methods. Routledge: New York.

Beaducel, A., \& Herzberg, P. (2006). On the performance of maximum likelihood versus means and variance adjusted weighted least squares estimation in CFA. Structural Equation Modeling, 13, 186-203. https://doi.org/10.1207/s15328007sem1302_2

Brown, T. (2006). Confirmatory factor analysis for applied research. New York: Guilford Press.

Byrne, B. (2008). Testing for multigroup equivalence of a measuring instrument: A walk through the process. Psicothema, 20(4), 872-882. Obtenido de: https://www.redalyc. org/pdf/727/72720455.pdf

Carrasco, I., Clemente, M., \& Llavona, L. (1989). Analisis del inventario de asercion de Gambrill y Richey. Estudios de psicologia, 37, 63-74. https://doi.org/10.1080/021093 $\underline{95.1989 .10821107}$

Castanyer, O. (1996). La Asertividad: expresion de una sana autostima. (3a Ed.) Bilbao Desclee de Brouwer.

Castaños, S., Reyes, I., Rivera, S., \& Díaz, R. (2011). Estandarización del inventario de Asertividad de Gambrill y Richey II. RIDEP, 1(29), 27-50. Obtenido de: https://www. redalyc.org/pdf/4596/459645441003.pdf

Cerquera, B. G. (2020). Comparación de la asertividad en estudiantes del primer y tercer grado de educación secundaria del distrito de Chorrillos. (Tesis de Maestría). Universidad Ricardo Palma. Lima. Perú. Disponible en: https://repositorio.urp.edu.pe/bitstream/handle/URP/3282/psic-T030_09486805_M\%20 $\% 20 \% 20 B L A N C A \% 20$ GIOVANNA\%20CERQUERA $\% 20$ HUAM $\%$ C $3 \% 81 \mathrm{~N}$. pdf? sequence $=1 \&$ isAllowed $=\mathrm{y}$

Chávez-Kanashiro, M., \& Garrido-Arévalo, S. (2015). Asertividad y asertividad sexual: Un análisis de relación en estudiantes universitarios de Lima Metropolitana. Revista Peruana de Obstetricia y Enfermería, 11(1). Disponible en: https://www.aulavirtualusmp.pe/ojs/index.php/rpoe/article/viewFile/738/577 
Chen, F. (2007). Sensitivity of Goodness of Fit Indexes to Lack of Measurement Invariance, Structural Equation Modeling: A Multidisciplinary Journal, 14(3), 464-504. https://doi.org/10.1080/10705510701301834

Cheung, G.W., \& Rensvold, R.B. (2002). Evaluating goodness-of-fit indexes for testing measurement invariance. Structural Equation Modeling, 9(2), 233-255.doi:10.1207/ s15328007sem0902_5

Colegio de psicólogos del Perú (2017). Código de ética y deontología. Recuperado de https://www.cpsp.pe/documentos/marco_legal/codigo_de_etica_y_deontologia. pdf?fbclid=IwAR20yCb5yoFa0d5-OGKXutIfnbVd7a_U-HwrE4b50--B3xooGQ39FFYt3Y

Coronel, C., Levin, M., \& Mejail, S. (2011). Las habilidades sociales en adolescentes tempranos de diferentes contextos socioeconómicos. Electronic Journal of Research in Educational Psychology, 9(1), 241-262. Obtenido de: http://www.investigacion-psicopedagogica.com/revista/articulos/23/english/Art_23_553.pdf

Cueva, M. R. C., Hernández, Y. L. D., \& Regalado, Ó. L. (2021). Comunicación asertiva en el contexto educativo: revisión sistemática. Revista Boletín Redipe, 10(4), 315-334. Disponible en: https://revista.redipe.org/index.php/1/article/download/1274/1180

Deng, L., \& Chan, W. (2017). Testing the difference between reliability coefficients alpha and omega. Educational and Psychological Measurement, 77(2), 185-203. https:// dx.doi.org/10.1177/0013164416658325

Escobar, J., \& Cuervo, A. (2008). Validez de contenido y juicio de expertos: una aproximación a su utilización: Avances en Medición, 6(1), 27-36. Recuperado de https:// www.researchgate.net/publication/302438451

Escurra, L. (1988). Cuantificación de la validez de contenido por criterio de jueces. Revista de Psicología, 6(1-2), 103-111. Recuperado de http://revistas.pucp.edu.pe/index. $\mathrm{php} / \mathrm{psicologia/article/view/4555}$

Espinoza, C., \& Panta, C. (2014). Estilos de socialización parental y asertividad en las estudiantes de cuarto año de secundaria de una institución educativa. Revista Cientifica-Facultad de humanidades, 5(1). Recuperado de: http://revistas.uss.edu.pe/index. php/PAIAN/article/view/226.

De Azevedo, J. (2016). The Asertiveness Guide For Woman. Oakland: New Harbinger.

Dimitrov, D.M. (2010). Testing for factorial invariance in the context of construct validation. Measurement and Evaluation in Counseling and Development, 43(2), 121-149. doi:10.1177/0748175610373459.

Domínguez Alonso, J., López Castedo, A., \& Álvarez Roales, E. (2015). Implicación de variables sociales y educativas en la conducta asertiva adolescente. Aula Abierta, 43(1), 26-31. doi:10.1016/j.aula.2014.05.004

Eslami, A. A., Rabiei, L., Afzali, S. M., Hamidizadeh, S., \& Masoudi, R. (2016). The Effectiveness of Assertiveness Training on the Levels of Stress, Anxiety, and Depression of High School Students. Iranian Red Crescent Medical Journal, 18(1). e21096. doi:10.5812/ircmj.21096 
Farley, J. P., \& Kim-Spoon, J. (2014). The development of adolescent self-regulation: Reviewing the role of parent, peer, friend and romantic relationships. Journal of Adolescence, 37(4), 433-440. https://doi.org/10.1016/j.adolescence.2014.03.009

Ferrando, P., \& Anguiano-Carrasco, C. (2010). El análisis factorial como técnica de investigación en psicología. Papeles del Psicólogo, 31(1), 18-33. Recuperado de: https:// www.redalyc.org/pdf/778/77812441003.pdf

Gaeta, L., \& Galvanovskis, A. (2009). Asertividad: un análisis teórico-empírico. Enseñanza e Investigación en Psicología, 14(2), 403-425. Recuperado de: https://www. redalyc.org/pdf/292/29211992013.pdf

Galassi, J., Hollandsworth, J., Radecki, J., Gay, M., Howe, M., \& Evans, C. (1976). Behavioral performance in the validation of an assertiveness scale. Behavior Therapy, 7(4), 447-452. https://doi.org/10.1016/S0005-7894(76)80163-3

Gambrill, E., \& Richey, C. (1975). An assertion inventory for use in assessment and research. Behavior Therapy, 6(4), 550-561. https://doi.org/10.1016/S0005-7894(75)80013-X

Garcia, E., \& Fabregas, J. (1988). Tecnica cognitiva de autocontrol. Madrid: Alhamb.

García-López, L. M., Díaz, D., González-Víllora, S., \& Valenzuela, A. (2012). Cambios en la empatía, la asertividad y las relaciones sociales por la aplicación del modelo de instrucción educacional deportiva. Revista de Psicología Del Deporte, 21(2), 321-330. Recuperado de: https://www.redalyc.org/pdf/2351/235126897012.pdf

González C., Guevara, Y., Jiménez, D., \& Alcázar, R. (2018). Relación entre asertividad, rendimiento académico y ansiedad en una muestra de estudiantes mexicanos de secundaria. Acta Colombiana de Psicologia, 21(1), 128-138. https://doi.org/10.14718/ ACP.2018.21.1.6

Gana, K., \& Broc, G. (2019). Structural Equation Modeling with Lavaan. New York: Wiley.

Guerra, M. (1996). Estandarización del inventario de Asertividad de Gambrill y Richey para población de la Ciudad de México.(Tesis de Pregrado).Universidad Nacional Autonoma de Mexico, México DF.

Hair, J.F., Black, W.C., Babin, B.J., \& Anderson, R.E. (2014). Multivariate Data Analysis. (7a Ed.). UK: Pearson.

Hu, L., \& Bentler, P. (1999). Cutoff criteria for fit indexes in covariance structure analysis: Conventional criteria versus new alternatives. Structural Equation Modeling, 6(1), 1-55. http://dx.doi.org/10.1080/10705519909540118

Laursen, B., \& Mooney, K. S. (2008). Relationship network quality: Adolescent adjustment and perceptions of relationships with parents and friends. American Journal of Orthopsychiatry, 78(1), 47-53. https://doi.org/10.1037/0002-9432.78.1.47

León, A., Rodríguez, C., \& Ferrel, F., \& Ceballos, G. (2009). Asertividad y autoestima en estudiantes de primer semestre de la Facultad de Ciencias de la Salud de una universidad pública de la ciudad de Santa Marta (Colombia). Psicología desde el Caribe, (24), 91-105. Recuperado de: http://rcientificas.uninorte.edu.co/index.php/psicologia/ article/download/615/328::pdf 
Luna, J., \& Ochoa, S. (2018). La comunicación en adolescentes con conductas disruptivas. La asertividad en adolescentes. Pensamiento Americano, 11(20), 43-52. https://doi. org/10.21803/pensam.v11i20.12

Milovanovic, R. (2021). Effects of an assertive behaviour program on the reduction of anxiety in future teachers and preschool teachers. Technium Social Science Journal, 17, 90-99. Disponible en: https://www.techniumscience.com/index.php/socialsciences/article/download/2707/1039

Milfont, T., \& Fischer, R. (2010). Testing measurement invariance across groups: Applications in cross-cultural research. International Journal of Psychological Research, 3(1), 111-121. Recuperado de: http://hdl.handle.net/10819/6503.

Millsap, R., \& Olivera-Aguilar, M. (2012). Investigating Measurement Invariance Using Confirmatory Factor Analysis. In R. Hoyle, (Ed.). Handbook of Structural Equation Modeling, (pp. 380-392). NY: The Guilford Press.

Moon, J. (2009). Academic assertiveness real life strategies for today's higher education students. New York: Routledge Taylor \& Francis group.

Morales, M., \& Díaz, D. (2018). Inventario de asertividad: propiedades psicométricas en adolescentes michoacanos, Integración Académica en Psicología, 6(18), 74 - 85. Obtenido de: http://integracionacademica.org/attachments/article/207/ Integraci $\% \mathrm{C} 3 \% \mathrm{~B} 3 \mathrm{n} \% 20 \mathrm{Acad} \% \mathrm{C} 3 \% \mathrm{~A} 9 \mathrm{mica} \% 20 \mathrm{en} \% 20 \mathrm{Psicolog} \% \mathrm{C} 3 \% \mathrm{ADa} \% 20$ V6N18.pdf\#page $=77$

Muñiz, J., Elosua, P., \& Hambleton, R. (2013). Directrices para la traducción y adaptación de los Tests: segunda edición. Psicothema, 25(2), 151-157. doi:10.7334/psicothema2013.24

Muthén, B., \& Kaplan, D. (1985). A comparison of some methodologies for the factor analysis of non-normal Likert variables. British Journal of Mathematical and Statistical Psychology, 38, 171-189.doi:10.5964/ijpr.v11i2.269.

Naranjo, M. (2008). Relaciones interpersonales adecuadas mediante una comunicación y conducta asertivas. Actualidades investigativas en Educación, 8(1), 1-27. Obtenido de: https://revistas.ucr.ac.cr/index.php/aie/article/download/9315/17783/

Navarro, G., Varas, M., \& Maluenda, J. (2015). Propiedades psicométricas del inventario de asertividad de gambrill y richey en estudiantes universitarios chilenos. Revista Iberoamericana de Diagnóstico y Evaluación, 1(43), 33-43. Obtenido de: https:// www.academia.edu/download/60714557/Art320190926-75108-6217h6.pdf

Núñez, C., Ramos-Vera, C.A., Serpa, A., \& Ogundokun, R.O. (2021). Adaptation of the Personality Type Inventory Based on Enneagram in Peruvian University Students of Health Sciences. Journal of Research in Medical and Dental Science, 9(5). https:// doi.org/10.13140/RG.2.2.33090.30404

Pailos, F. (2014). El comportamiento de la verdad y de la justificación y su relación con la práctica asertiva. Revista de Filosofía, 70, 119-131. http://dx.doi.org/10.4067/S0718$\underline{43602014000100008}$ 
Parray, W. M., Kumar, S., David, B. E., Khare, S., \& Maurya, V. P. (2020). Exploring assertiveness in Indian adolescents: A qualitative inquiry. Journal of Indian Association for Child \& Adolescent Mental Health, 16(2), 106-122. Obtenido de: http:// web.a.ebscohost.com/ehost/pdfviewer/pdfviewer?vid=0\&sid=0ecd9e37-296c-44829f46-2e28770dce01\%40sessionmgr4006

Peneva, I., \& Mavrodiev, S. (2013). A historical approach to assertiveness. Psychological Thought, 6(2008), 3-26. doi:10.5964/psyct.v6i1.14

Plenty, S., Östberg, V., Almquist, Y. U., Augustine, L., \& Modin, B. (2014). Psychosocial working conditions: An analysis of emotional symptoms and conduct problems amongst adolescent students. Journal of Adolescence, 37(4), 407-417. https://doi. org/10.1016/j.adolescence.2014.03.008

Postolatii, E. (2017). Assertiveness: Theoretical approaches and benefits of assertive behavior. Journal of Innovation in Psychology, Education and Didactics, 21(1), 83-96. http://www.jiped.ub.ro/wp-content/uploads/2017/09/JIPED_21_1_2017_7.pdf

Ramos-Vera, C. (2020a). Un método de cálculo del tamaño muestral en modelos de ecuaciones estructurales. Revista Perspectiva Empresarial, 7(2), 7-9. https://doi. org/10.16967/23898186.648

Ramos-Vera, C. A. (2020b). Replicación bayesiana: cuán probable es la hipótesis nula e hipótesis alterna. Educación Médica. https://doi.org/10.1016/j.edumed.2020.09.014

Ramos-Vera, C.A. (2021a). The Bayes Factor, a Suitable Complement beyond Values of p $<0.05$ in Nursing Research and Education. Investigacion y Educación en Enfermería, 39(1): e14. https://doi.org/10.17533/udea.iee.v39n1e14

Ramos-Vera, C.A. (2021b). The use of Bayes factor in clinical cardiology research. Revista Española de Cardiología, 74(7), 641-642. https://doi.org/10.1016/j.rec.2021.01.020

Ramos-Vera, C. (2021c). Las redes de relación estadística en la investigación psiquiátrica: el caso del delirio en el contexto de COVID-19. Revista Colombiana de Psiquiatría. https://doi.org/10.1016/j.rcp.2021.02.004

Ramos-Vera, C.A., Dávila, A., Castañeda, J., Ramírez, Y., \& Serpa, A. (2021). Estructura e invarianza factorial de una escala de perfeccionismo infantil adaptada para adolescentes. Revista de Psicopatología y Psicología Clínica, 26(2), 141-153. https://doi. org/10.5944/rppc.27301

Ramos-Vera, C.A., Ramírez, Y., Rojas., E., Serpa, A., \& García, FE. (2021). Evidencias psicométricas mediante SEM y análisis de red del Inventario de Crecimiento Postraumático en adolescentes peruanos. Psicologia Conductual, 29(2). DOI: 10.13140/ RG.2.2.28892.72323

Ramos-Vera, C.A., \& Serpa, A. (2021). Network analysis in psychiatric research. Actas Españolas de Psiquiatria. 49(3), 123-124.

Ramos-Vera, C. A., Serpa, A., \& Lemos, V. (2020). Estructura factorial de la Escala de Personalidad Creadora (EPC) en adolescentes peruanos: invariancia de medida según el sexo. Asociación para el Desarrollo de la Ciencia y la Tecnología; Espacios, 41(18), 1-11. https://www.revistaespacios.com/a20v41n18/a20v41n18p22.pdf 
Rodríguez, M., \& Ruiz, M. (2008). Atenuación de la asimetría y de la curtosis de las puntuaciones observadas mediante transformaciones de variables: Incidencia sobre la estructura factorial. Psicológica, 29(2), 205-227. Obtenido de: https://dialnet.unirioja.es/servlet/articulo?codigo $=2718372 \&$ orden $=166859 \&$ info $=$ link

Salazar, I., Roldán, G., Garrido, L., \& Parejo, J. (2014). La asertividad y su relación con los problemas emocionales y el desgaste en profesionales sanitarios. Psicología Conductual, 22(3), 523-549. Obtenido de: https://search.proquest.com/docview/16441500 75 ?accountid $=37408$

Saldaña, G., Contreras, M., \& Albornoz, J. (2017). Propiedades psicométricas del inventario de asertividad de gambrill y richey en estudiantes universitarios chilenos. Revista Iberoamericana de Diagnóstico y Evaluación Psicologica, 43(1), 33-43. Recuperado de: https://www.academia.edu/download/60714557/Art320190926-75108-6217h6.pdf

Sarmiento, S. E., González, J. L., Hermida, R. U., Celis, H. A. C., \& Nieves, I. E. (2021). Asertividad: diferencias de género en estudiantes de nuevo ingreso en psicología en una universidad pública. Revista Electrónica del Desarrollo Humano para la Innovación Social, 8(15). Disponible en: https://cdhis.org.mx/index.php/CAGI/article/ view/159

Sifuentes, B. (2000). Estudio Psicométrico del Inventario de Aserción de E.D Gambrill y Ch. A. Richey, en Estudiantes Universitarios de Psicología (Tesis de maestria). Lima, Perú.

Sitota, G. (2018). Assertiveness and academic achievement motivation of adolescent students in selected secondary schools of Harari peoples regional state, Ethiopia. International Journal of Education and Literacy Studies, 6(4), 40-46. http://dx.doi. org/10.7575/aiac.ijels.v.6n.4p.40

Sodikin, M. A., Keliat, B. A., \& Wardani, I. Y. (2021). The effects of cognitive behaviour therapy and assertiveness training in chronic low self-esteem clients. Enfermería Clínica, 31, S96-S99. https://doi.org/10.1016/j.enfcli.2020.09.007

Soper, D.S. (2020). A-priori sample size calculator for structural equation models [Software]. Disponible en: https://www.danielsoper.com/statcalc

Tatari, F., Farnia, V., Momeni, K., Davarinejad, O., Salemi, S., Soltani, B., ... \& Alikhani, M. (2020). Predicting addiction potential based on sensation-seeking, psychological hardiness and assertiveness in students in western Iran: an analytical study. Journal of Substance Use, 1-6. https://doi.org/10.1080/14659891.2020.1760371

Thompson, R., \& Berenbaum, H. (2011). Adaptive and aggressive assertiveness scales (AAA-S). Journal of Psychopathology and Behavioral Assessment, 33(3), 323-334. Doi: 10.1007/s10862-011-9226-9

Vagos, P., \& Pereira, A. (2009). Qualitative analysis on the concept of assertiveness. In K. A. Fanti (Ed.), Applying psychological research to understand and promote the wellbeing of clinical and non-clinical populations (pp. 99-112). Athens, Greece: Atiner.

Vagos, P., \& Pereira, A. (2010). A proposal for evaluating cognition in assertiveness. Psychological Assessment, 22(3), 657-665. https://doi.org/10.1037/a0019782 
Vagos, P., \& Pereira, A. (2016). A cognitive perspective for understanding and training assertiveness. European Psychologist. 21, 109-121. https://doi.org/10.1027/1016-9040/ $\underline{\mathrm{a} 000250}$

Vagos, P., \& Pereira, A. (2018). Towards a cognitive-behavioral understanding of assertiveness: effects of cognition and distress on different expressions of assertive behavior. Journal of Rational-Emotive \& Cognitive-Behavior Therapy. 37, 133-148. https://doi.org/10.1007/s10942-018-0296-4

Vagos, P., \& Pereira, A. (2020). Revisiting a taxonomy of social anxiety and assertiveness in adolescence: evidence for a cognitive approach. Current Psychology, 1-10. https:// doi.org/10.1007/s12144-020-00823-Z

Wolpe, J. (1973). Assertive training. In J. Wolpe (Ed.), The practice of behavior therapy (2da ed.) (pp. 80-94). Oxford, England: Pergamon Press. 